The Astrophysical Journal, 339:603-618, 1989 April 15

(C) 1989. The American Astronomical Society. All rights reserved. Printed in U.S.A.

\title{
EVOLUTION OF THE INTERGALACTIC MEDIUM IN A COLD DARK MATTER-DOMINATED UNIVERSE
}

\author{
Wei-Hwan Chiang, Dongsu Ryu, and Ethan T. Vishniac ${ }^{1}$ \\ Department of Astronomy, University of Texas \\ Received 1988 April 25 ; accepted 1988 September 12
}

\begin{abstract}
We study the evolution of the intergalactic medium (IGM) in a cold dark matter-dominated universe. The dark matter is approximated as a pressureless ideal fluid. The IGM is approximated as an ordinary fluid. Several three-dimensional numerical simulations are performed to follow the evolution of the model universe from $z \sim 100$ to the present time. Galaxies are assumed to form from $z=10$ to $z=1$ at the highest density peaks. This leads to a burst of galaxy formation. They are subsequently assumed to release energy back into the IGM over a period of $10^{8} \mathrm{yr}$. The IGM is allowed to cool by Compton and radiative cooling. The model simulates a comoving volume of $\left(9.6 h^{-1}\right)^{3} \mathrm{Mpc}^{3}$ in current units on a grid of $32^{3}$ cells for $h=1$ and $h=0.5$.

The major results are the following: (1) The growth of the root mean square density fluctuations in the IGM is greatly reduced during the period of heating, but it picks up at late times as gravity dominates again. (2) The density contour plots show large connected structure in the IGM. (3) The power spectrum for the IGM on smaller scales grows slower than the power spectrum of the dark matter. The slope of the two-point correlation function for the IGM is similar to that of the observed galaxy-galaxy correlation function between separations $1 h^{-1} \mathrm{Mpc}<r<3 h^{-1} \mathrm{Mpc}$. (4) The amount of gas with temperatures below $10^{4} \mathrm{~K}$ is negligible, that between $10^{4}$ and $10^{5} \mathrm{~K}$ is $\sim 10 \%-20 \%$, that between $10^{5}$ and $10^{6} \mathrm{~K}$ is $\geq 40 \%$, and that above $10^{6} \mathrm{~K}$ is $\sim 40 \%$. The fraction of neutral hydrogen is very small. (5) The variation in pressure is $\sim 10$ times that in the temperature, suggesting no pressure equilibrium. Pressure is generally higher in regions of higher densities. (6) Optically thin lines computed show a variety of profiles. (7) The IGM has peculiar velocities of $\sim 150 \mathrm{~km} \mathrm{~s}$. The possible relevance of the model results to the forest of the Ly $\alpha$ absorption lines observed in quasars is discussed.
\end{abstract}

Subject headings: cosmology — dark matter — galaxies: intergalactic medium

\section{INTRODUCTION}

In recent years, considerable progress has been made in the attempt to explain the origin of the large-scale structure of the universe. To date the most extensively explored, and probably the most successful, model is the one dominated by weakly interacting particles with low velocity dispersions (cold dark matter [CDM]), such as axions and photinos (e.g., Blumenthal et al. 1984). For a recent review see the paper by White (1986). In such a model universe, the amount of baryonic matter is constrained by the standard theory of primordial nucleosynthesis, which places an upper limit for baryonic matter at $\Omega_{B} \sim 0.12$. The initial density fluctuation power spectrum is assumed to have the Zel'dovich-Harrison form $\left|\delta_{k}\right|^{2} \propto k$, and the development of large-scale structures comes entirely from gravitational amplification of the initial density fluctuation. Its nonlinear growth has been studied extensively via $N$-body simulations (Melott et al. 1983; Davis et al. 1985; White et al. 1987), and also by Peebles (1987) and Ryu and Vishniac (1988) using a fluid approximation. Since these studies consider only the dark matter, in order to link the model distribution of the dark matter to the observed galaxy distribution one must make assumptions concerning the relation between the two. Under the hypothesis that galaxies form only at high-density peaks of dark matter distribution (biased galaxy formation; Kaiser 1984, 1986) the results of the CDM models can be made to match the observations reasonably well, even though the status of real physical mechanisms giving rise to biasing is at present ambiguous (e.g., Dekel and Rees 1987).

${ }^{1}$ Alfred P. Sloan Foundation Fellow and Presidential Young Investigator.
Taking the view that galaxies formed out of baryonic matter in the early universe, one might expect that some portion of this matter would have been left as an intergalactic medium (IGM), unless the efficiency of formation proceeded at the improbable $100 \%$ level. In fact, there are observations indicating the existence of an IGM. The most direct source of information is provided by the Ly $\alpha$ absorption lines identified by Lynds (1971) in high-redshift quasar absorption-line spectra. These clouds were subsequently studied in greater detail and interpreted by Sargent et al. (1980) as arising from absorptions by cosmologically distributed intervening material. They proposed that the Ly $\alpha$ clouds (density $\sim 10^{-4}$ to $4 \times 10^{-3} \mathrm{~cm}^{-3}$, temperature $\sim 3 \times 10^{4} \mathrm{~K}$, and diameter $10^{20}-10^{23} \mathrm{~cm}$ ) were photoionized by the integrated quasar light and were pressureconfined by a hotter and less dense background medium (density $\sim 10^{-5} \mathrm{~cm}^{-3}$, temperature $\sim 3 \times 10^{5} \mathrm{~K}$ ). On the other hand, the confinement of intergalactic clouds by gravity has been suggested by Black (1981), Ikeuchi and Ostriker (1986), and Rees (1986), and the possibility that they may be expanding cannot be ruled out. The generation of intergalactic clouds caused by explosions of pregalactic objects has been studied by Ostriker and Ikeuchi (1983), Vishniac, Ostriker, and Bertschinger (1985), Ikeuchi and Ostriker (1986), and Vishniac and Bust (1987). The dense shells produced in explosions may fragment as a result of a dynamic instability (Vishniac 1983), if the shell is not too massive. The fragments can be interpreted as the Ly $\alpha$ clouds. A different picture was put forward by Hogan (1987), who argued that Ly $\alpha$ absorption lines might arise from a much more dense environment. Despite the differences, it is generally agreed that the $\operatorname{Ly} \alpha$ clouds are objects 
produced early in the universe and constitute an important component of the IGM. Finally, a study of the correlation of quasar absorption lines in a CDM-dominated universe was made by Salmon and Hogan (1986), under the assumption that the distribution of the absorption material is the same as that of the CDM.

The present study is motivated by the progress made in the aforementioned two areas. We wish to consider the dark matter and the baryons together, since they are coupled gravitationally. Specifically, we ask the following question: how would the baryonic matter left over from the galaxy formation evolve in a CDM-dominated universe if there is significant energy feedback from the pregalactic objects? Since some of the detailed physical processes involved are not yet completely understood, we adopt several simple assumptions in order to make further progress. Our results should be read with these and other restrictions (see below) in mind. In this paper, § II describes the basic assumptions of the model and the numerical method of solving the basic equations, $\S$ III presents the results of numerical simulations, and $\S$ IV gives our discussion and the conclusions.

\section{THE MODEL}

\section{a) The Basic Equations and Assumptions}

We consider a flat model universe that is initially composed of two components - cold dark matter and baryonic matter (IGM). The dark matter constitutes $90 \%$ of the mass $\left(\Omega_{\mathrm{CDM}}=\right.$ $0.9)$, whereas the baryons constitute $10 \%\left(\Omega_{\mathrm{IGM}}=0.1\right)$. The IGM is assumed to be composed of hydrogen ( $75 \%$ by mass) and helium ( $25 \%$ by mass). The model describes the evolution of such a universe from an instant corresponding to redshift $z \sim 100$ to the present epoch in the Newtonian approximation. We allow galaxies to form during the period $z=10$ to $z=1$, and subsequently inject energy back into the IGM (see below). The word "galaxy" here should, however, be interpreted broadly as any object formed that injects a significant amount of energy back into the IGM.

The CDM component is approximated as a pressureless fluid and the IGM component as an ordinary fluid. We adopt $L$ (length) $\equiv \Delta x, T$ (time) $\equiv t_{0}$, and $D$ (density) $\equiv \bar{\rho}=$ $\left(3 \dot{a}^{2} / 8 \pi G a^{2}\right)$ as our three basic units of scaling, where $a$ is the scale factor of the expanding universe, $\Delta x$ and $t_{0}$ are arbitrary length and time units to be chosen $(\Delta x$ is chosen to correspond to the comoving cell size and $t_{0}$ the age of the universe in the numerical computation), and $\bar{\rho}$ is the average density of the universe. The basic equations for the model, written in the comoving frame, become

$$
\begin{gathered}
\frac{\partial \rho_{d}}{\partial t}+\nabla \cdot\left(\rho_{d} v\right)=0 \\
\frac{\partial v}{\partial t}+(v \cdot \nabla) v+\frac{4}{3 t} v+\nabla \Phi=0 \\
\frac{\partial \rho_{\mathrm{IGM}}}{\partial t}+\nabla \cdot\left(\rho_{\mathrm{IGM}} u\right)=0 \\
\frac{\partial u}{\partial t}+(\boldsymbol{u} \cdot \nabla) \boldsymbol{u}+\frac{4}{3 t} u+\nabla \Phi+\frac{1}{\rho_{\mathrm{IGM}}} \nabla p=0 \\
\frac{\partial t}{\boldsymbol{u}}+(\boldsymbol{u}) e+\frac{4}{3 t} e+\frac{p}{\rho_{\mathrm{IGM}}}(\nabla \cdot u)+\frac{2}{t} \frac{p}{\rho_{\mathrm{IGM}}}=\frac{1}{\rho_{\mathrm{IGM}}}(\Gamma-\Lambda)
\end{gathered}
$$

$$
\nabla^{2} \Phi=\frac{2}{3 t^{2}}\left(\rho_{d}+\rho_{\mathrm{IGM}}-1\right)
$$

Here we have used the following notations: $\rho_{d}=$ density of CDM, $\rho_{\mathrm{IGM}}=$ density of IGM, $v=$ velocity of CDM, $\boldsymbol{u}=$ velocity of IGM, $p=$ pressure of IGM, $e=$ internal energy of IGM per unit mass, $\Phi=$ gravitational potential, $\Gamma=$ heating function, and $\Lambda=$ cooling function. Equations (1) and (3) state the conservation of mass for the CDM and the IGM, respectively. Equations (2) and (4) give the conservation of momentum for the CDM and the IGM. Equation (5) gives the conservation of energy for the IGM. Equation (6) is the Poisson's equation.

The heating term depends on the history of galaxy formation and the process of energy injection associated with individual galaxies. Since the detailed physics involved is not fully understood, we shall adopt a simple yet plausible rule (see below). For the cooling term, we use the equilibrium cooling curve for a pure hydrogen and helium plasma calculated by Vishniac and Bust (1987) in conjunction with a redshift-dependent Compton cooling formula. We have assumed that the total mass is conserved for both the CDM and the IGM separately. That is to say, as galaxies form in the model, no reduction of mass in the IGM is taken into account. This is probably a reasonable simplification for a first study, and the results are not expected to be sensitive to it. The efficiency for galaxy formation is not known. We do not follow the motion of galaxies, partly to save computational time and partly because our calculation is too crude to supply the initial velocity of the galaxy. Such a simplification is acceptable if the distance traversed by individual galaxies during the energy injection period is sufficiently small. For example, if the peculiar velocity of a galaxy at birth is $500 \mathrm{~km} \mathrm{~s}^{-1}$, it will have traversed only $50 \mathrm{kpc}$ in $10^{8} \mathrm{yr}$. This distance is far smaller than the computational cell size at the present epoch, which is $0.3 h^{-1} \mathrm{Mpc}$.

There are two reasons why a pressureless fluid approximation for the dark matter may have difficulties. First, if in a volume element several streams of microscopic particles move in different directions, then a single mean velocity is no longer adequate. This may occur when collisionless particles cross one another, associated with the formation of caustics. Second, particles may acquire random velocities as a result of the gravitational interaction between individual particles and the rapid varying gravitational potential produced by the particles partaking in the contraction. Both of these processes may occur in the highly nonlinear regime of the evolution. The structure of the caustics will, in any case, be smoothed out by the artificial viscosity present in the numerical code, whereas the pressure effect will be mimicked by the grid resolution, since the cell size is the lower limit to which a fluid parcel may be compressed. Quantitative estimates of the errors that arise from these two effects are difficult to make.

Our major objective is to study the evolution of the IGM in such a model universe. Since the basic equations are analytically intractable, we will solve them numerically.

\section{b) The Numerical Procedure}

The numerical program simulates a finite volume of the universe that has a cubic shape. The physical size of the cube grows according to the expansion parameter $a$, and corresponds to $\left(9.6 h^{-1}\right)^{3} \mathrm{Mpc}^{3}$ at the present epoch, where $h$ is the Hubble constant at the present time in units of $100 \mathrm{~km} \mathrm{~s}^{-1} \mathrm{Mpc}^{-1}$. This fundamental cube is subdivided into 
$32^{3}$ cells; within each cell all relevant physical variables are assigned either initially or at subsequent moments and are followed up to the present epoch. Periodic boundary conditions are adopted.

The initial condition (corresponding to a time at redshift of $\sim 100$ ) for a given model run is determined as follows. The density fluctuation for the CDM is assumed to be a Gaussian random field with the power spectrum $\left|\delta_{k}\right|^{2}=A k /(1+\alpha k$ $\left.+\beta k^{1.5}+\gamma k^{2}\right)^{2}$, where $\delta$ is the usual dimensionless density contrast and $\boldsymbol{A}$ is a normalization constant (Davis et al. 1985). If $k$ is expressed in terms of comoving length unit, the parameters are $\alpha=1.7 q, \quad \beta=9.0 q^{1.5}$, and $\gamma=1.0 q^{2}$, where $q=\left(\Omega h^{2} \theta^{-2}\right)^{-1} \mathrm{Mpc}$ and $\theta$ is the temperature of the microwave background radiation in units of $2.7 \mathrm{~K}$. The magnitude of the initial density fluctuation is determined by an extrapolation backward in time based on observations of the present universe (Peebles 1982). The initial momentum is calculated from equation (2) using a linear approximation. The same initial spectrum is also used for the IGM. In addition, the IGM is assumed to have a negligible amount of thermal energy at the beginning. Knowing the initial distribution of the matter, the gravitational potential can be computed by solving the Poisson's equation using the method of fast Fourier transforms. For the sake of simplicity, we do not update the gravitational potential at every step; instead we use the linear theory to extrapolate the gravitational potential at subsequent times. Although this method is crude, it is a reasonable first approximation, since a gravitational potential perturbation does not evolve significantly until the corresponding density perturbation is extremely nonlinear.

The model universe then evolves forward in time according to the model equations (1)-(6) by employing a combination of first-order finite-difference equations and a beam scheme method (Sanders and Prendergast 1974). The equilibrium radiative cooling and the Compton cooling are included throughout the model computation, the cooling time scale for the latter being $\left(240 \times 10^{10}\right) /(1+z)^{4}$ yr. Galaxies are allowed to form during the period between $z=10$ and $z=1$. A galaxy forms if the gas density in a given cell becomes higher than a critical value; moreover, it is assumed that no two galaxies are formed in the same cell during a given model run. The critical density (5-6 times the rms density fluctuation of one cell size) for galaxy formation is chosen such that the total number of galaxies formed in a model computation is approximately between 800 and 900 . This corresponds to an individual galaxy collecting all the material in a volume $\sim 1 \mathrm{Mpc}^{3}$ in the present units. Each galaxy is assumed to release $10^{60}$ ergs of energy into the IGM over $10^{8} \mathrm{yr}$ locally, i.e., in the same cell in which a galaxy is born, since we do not follow the motions of galaxies. Also, $10^{60}$ ergs is approximately the amount of the energy released by an individual quasar (Weedman 1986). Computa- tionally the heating process is modeled by raising the thermal energy of the gas in the cell where a galaxy resides. We note that this particular criterion for galaxy formation leads to a burst of galaxy formation between $z=10$ and $z=6$ (see Fig. 2). On the other hand, preliminary results indicate that the state of the model IGM at the present epoch is very similar to that produced in a model for which the threshold density of galaxy formation is set by the ratio between the local IGM density and the mean IGM density.

The complete procedure for a given computer model run consists of (1) setting up the initial conditions for both the CDM and the IGM; (2) selecting the time step according to a modified Courant condition and the cooling time scale; (3) transporting both fluids according to the method of beam scheme; (4) updating the thermal energy content as a result of heating and cooling, and momentum and momentum flux arising from work done by gravity and by the fictitious force present in the comoving frame; (5) marking the cells of galaxy formation if the redshift is in the right interval and if the density is high enough; and (6) repeating the second step to complete the cycle.

The numerical code was tested with a variety of simplified cases for which analytic solutions may be calculated. The portions for setting up the initial conditions and that using the beam scheme were separately tested and previously used in solving other problems (Chiang and Prendergast 1985; Ryu and Vishniac 1988).

\section{NUMERICAL RESULTS}

Two models are computed in this study, the first having $h=1$ and the second $h=0.5$. For each model, four computer runs corresponding to different realizations of the initial conditions are performed for the purpose of calculating statistical quantities. The parameters for each model are given in Table 1.

\section{a) Growth of Density Fluctuation}

Figure 1 displays the evolution of the rms density fluctuations of one-cell size of the IGM for both models. The broken curve represents the averages over four runs for each model, whereas the solid line gives the linear prediction. Initially the growth of the IGM almost parallels that of the linear prediction, with a slightly lower amplitude because the gas pressure tends to work against the gravity, which is the only force responsible for the growth. The growth of fluctuations in the IGM becomes very depressed during the period between $z=10$ and $z=5$. Afterward, the growth approximately parallels that of the linear prediction. Figure 2 shows the amount of heating as a function of time. We note that as soon as the galaxy formation epoch is reached, galaxies form rapidly, since many cells are already very dense. The depression in the growth of fluctuations in the IGM between $z=10$ and $z=5$

TABLE 1

Model Parameters

\begin{tabular}{cccccccccccc}
\hline \hline Model & $h$ & $\Omega_{\text {CDM }}$ & $\Omega_{\text {IGM }}$ & $t_{\text {init }}$ & $\delta_{\text {init }}$ & $\begin{array}{c}\tau_{\text {univ }} \\
(\mathrm{yr})\end{array}$ & $\begin{array}{c}E_{\text {gal }} \\
(\mathrm{ergs})\end{array}$ & $\begin{array}{c}\tau_{\text {gal }} \\
(\mathrm{yr})\end{array}$ & $\begin{array}{c}\Delta z \\
\rho_{\text {crit }}\end{array}$ & $\begin{array}{c}\text { Number } \\
\text { of Runs }\end{array}$ \\
\hline $1 \ldots \ldots \ldots$ & 1 & 0.9 & 0.1 & $3.54 \times 10^{-4}$ & 0.1 & $10^{10} h^{-1}$ & $10^{60}$ & $10^{8}$ & $10-1$ & 6 & $0.3 h^{-1}$ \\
$2 \ldots \ldots \ldots$ & 0.5 & 0.9 & 0.1 & $3.54 \times 10^{-4}$ & 0.1 & $10^{10} h^{-1}$ & $10^{60}$ & $10^{8}$ & $10-1$ & 5 & $0.3 h^{-1}$ \\
\hline
\end{tabular}

NoTE. $-t_{\text {init }}$ is the initial time in units of $\tau_{\text {univ }}$, the age of the universe; $\delta_{\text {init }}$ is the initial rms density fluctuations; $E_{\text {gal }}$ is the energy released per galaxy over the period $\tau_{\text {gal }} ; \Delta z$ is the redshift interval during which period galaxies form; $\rho_{\text {crit }}$ is the threshold density for galaxy formation, which corresponds to 5 or 6 times the rms density fluctuation; $\Delta x$ is the cell size in the computational grid. 


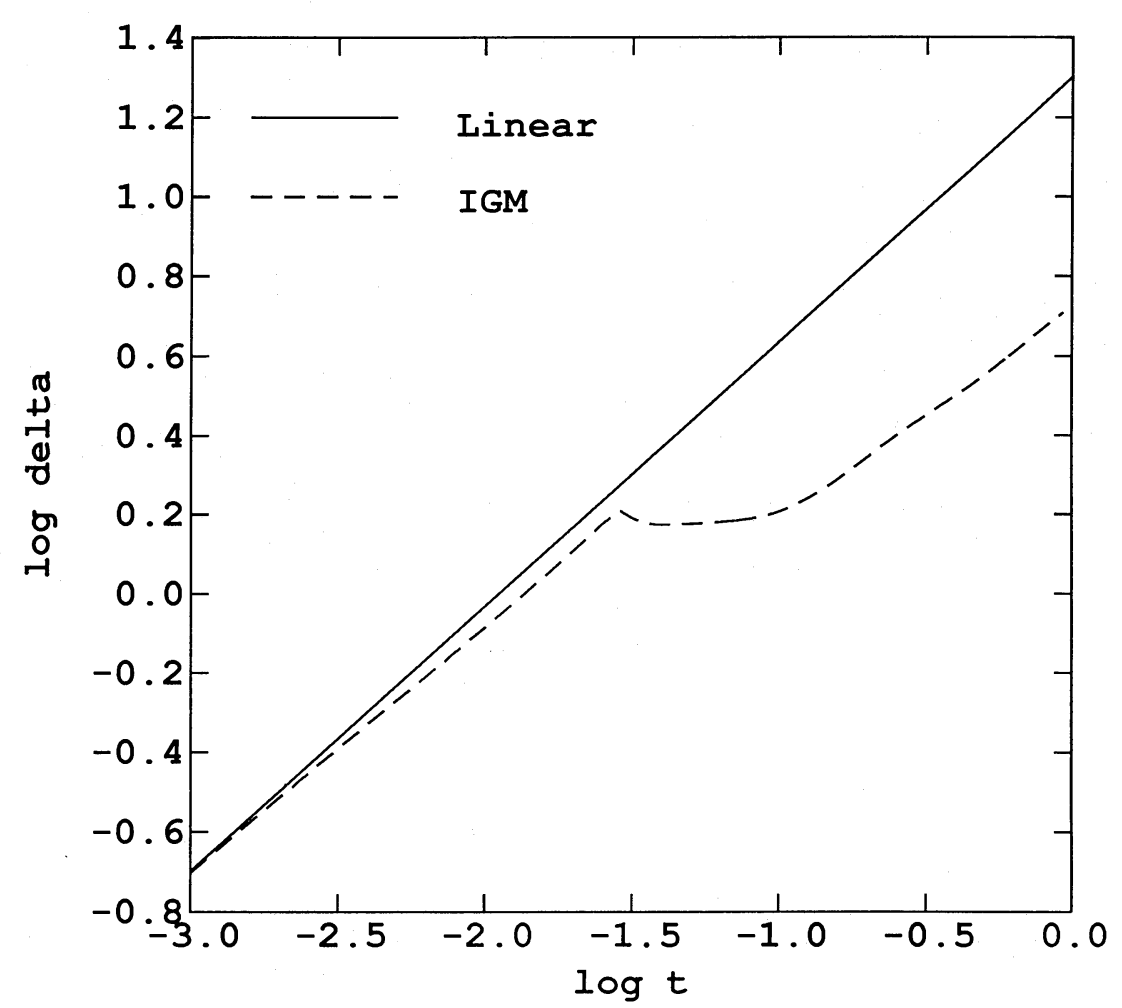

FIG. $1 a$

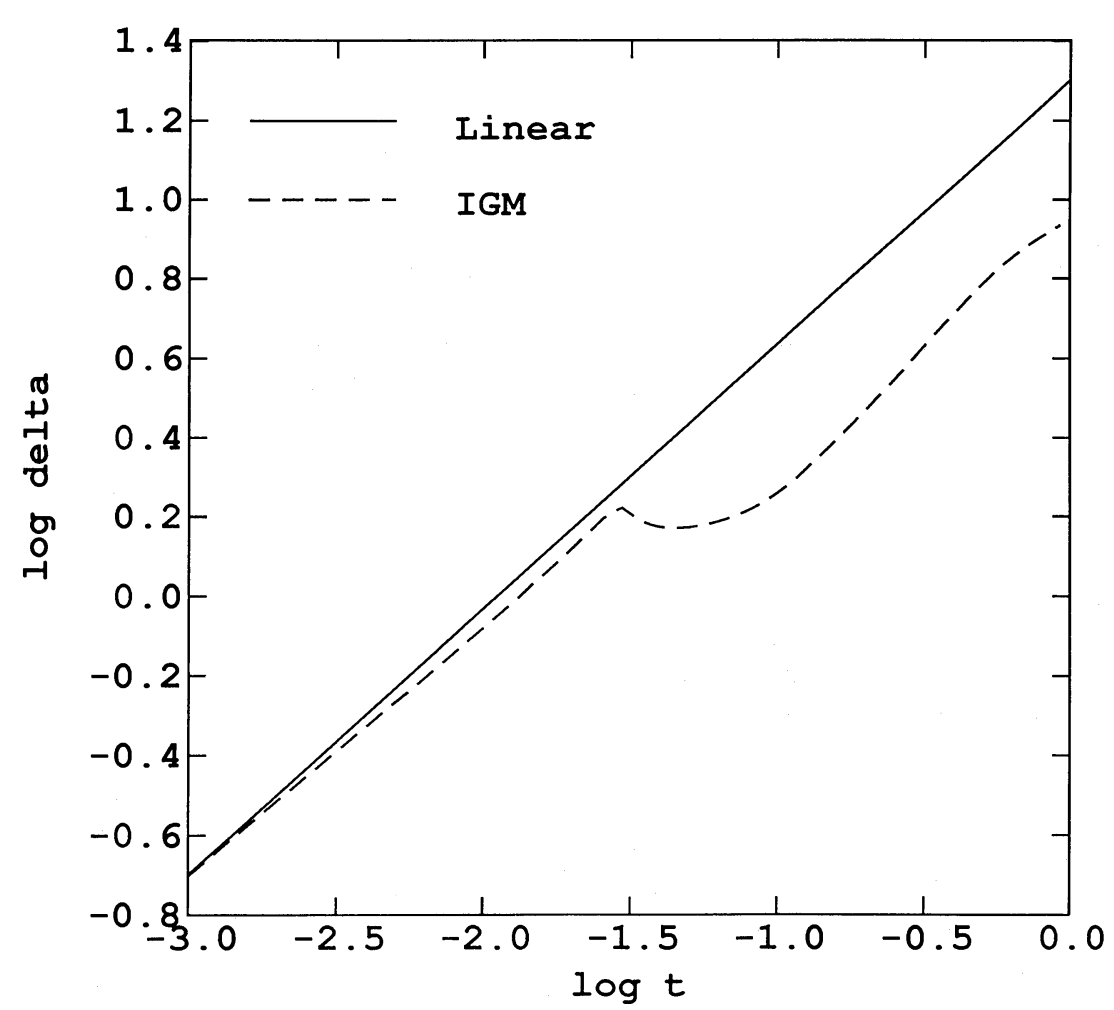

FIG. $1 b$

Fig. 1.-Root mean square density fluctuation in one-cell size, plotted as a function of time, where $t=1$ corresponds to the present epoch. The solid line

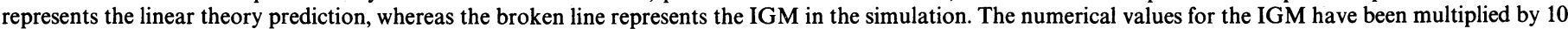
to facilitate the plot. $(a)$ model $1(h=1) ;(b)$ model $2(h=0.5)$. Note the substantially higher growth in model 2.

606 


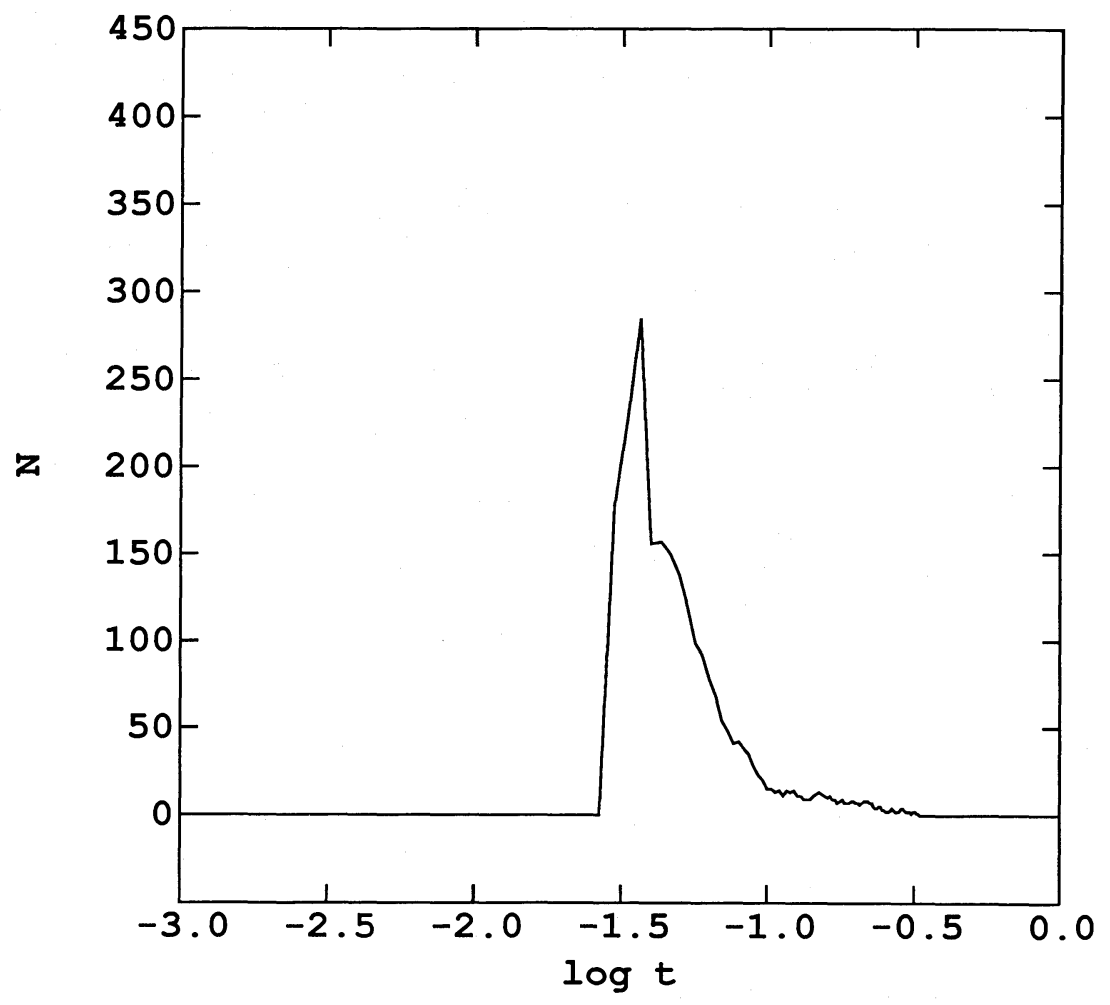

FIG. $2 a$

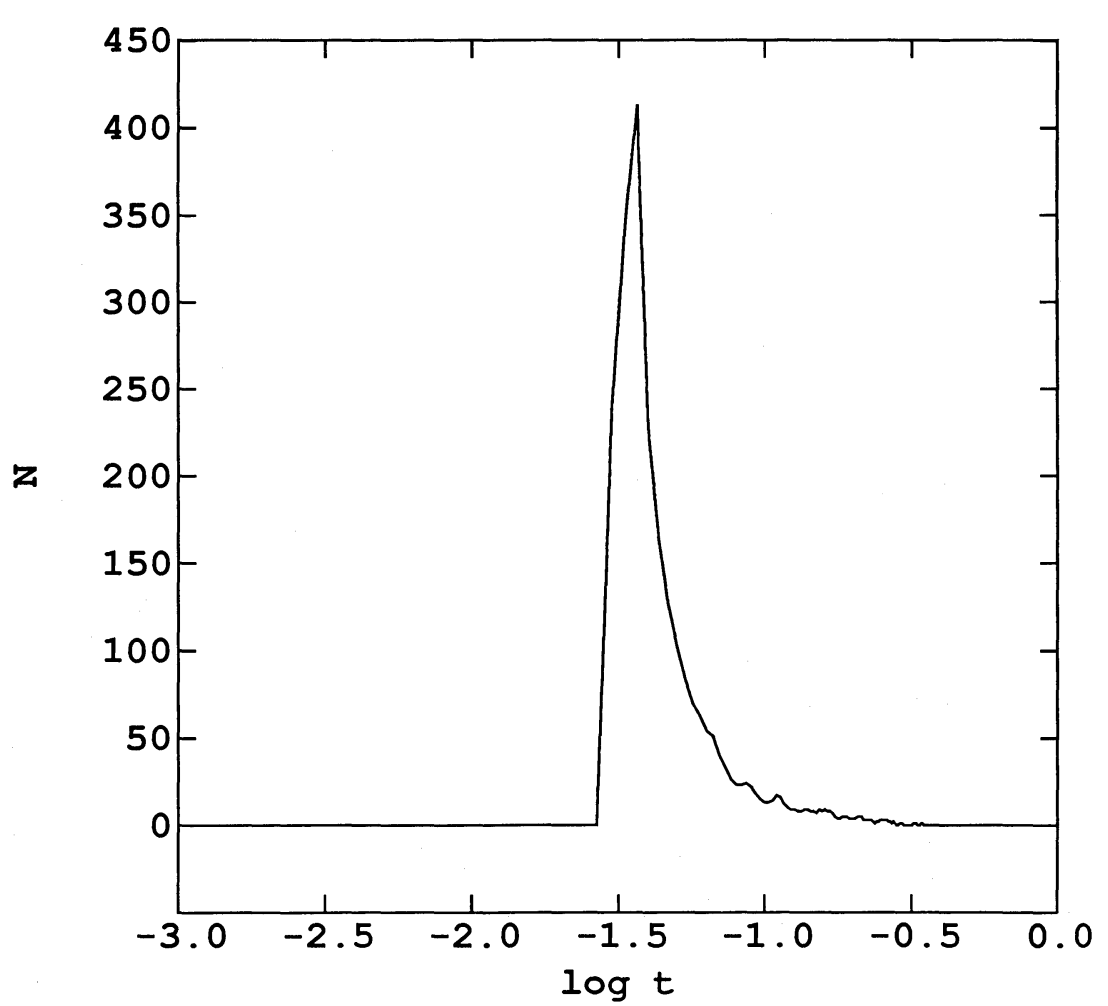

FIG. $2 b$

FIG. 2.-Number of active galaxies, (i.e., galaxies providing heating) plotted as a function of time: (a) model 1; (b) model 2 . The exceedingly sharp rise in the curve at $\log t \sim-1.5$ corresponds to the epoch of the onset of galaxy formation. The areas under the curves are about the same because approximately equal numbers of galaxies are formed in the two models.

607 
means that galaxy heating has led to a temporary expansion of gas away from local gravitational potential minimum. As the effect of heating diminishes, gravity takes over again.

Qualitatively the growth of density fluctuations is similar for the two models. However the growth in model 2 is slightly higher for two reasons: (1) the initial power spectra are slightly different in the two models, model 2 having more power on larger scales (see Fig. 7); (2) the effect of galaxy heating is reduced, since the same amount of heating energy is distributed in a volume 8 times as large. At the present epoch, the rms density fluctuation of IGM in model 2 is $\sim 30 \%$ higher than that in model 1.

\section{b) Overall Morphology of Density Distribution}

Different runs of the same model show the same qualitative behavior. As an example, we select the fourth run of model 1 for illustration. We should bear in mind that the topology associated with these graphs depends on the density level used. Figure 3 shows the distribution of the model IGM at two epochs $(z=1$ and $z=0)$; the surfaces correspond to a density level twice that of the mean. No smoothing is employed in making these plots. Recall that $z=1$ is the latest epoch for model galaxy formation, and by that time the heating from galaxies has nearly ended.

The primary features shown in these plots are the following: (1) The volume containing the model IGM with densities higher than twice the mean shrinks continually from $z=1$ to $z=0$. Were the simulation to continue, the distribution of matter would probably be concentrated in a few massive clumps. (2) The distribution is dominated by large connected structures. Heating from galaxies tends to disperse gas, and small clumps of dark matter (i.e., regions of local potential minimum) will not be able to confine gas. Similar plots of run 4 of model 2 (which uses the same seed number to generate the initial condition) are very similar to those in Figure 3, but quantitative differences do exist (see § III e).

\section{c) Temperature Distribution}

The overall temperature structure of the model IGM can be partially revealed by calculating the cumulative volume filling factor and the mass fraction as a function of temperature. Figure 4 displays these quantities for both models at $z=1$ and $z=0$, respectively. In both models the amount of gas with temperatures below $10^{4} \mathrm{~K}$ and its corresponding volume filling factors are less than $1 \%$, indicating a very small fraction of neutral hydrogen. The volume filling factor for gas hotter than $10^{6} \mathrm{~K}$ increases roughly by a factor of $2-3$ from $z=1$ to $z=0$ (from $\sim 20 \%$ to $\sim 40 \%$ in model 1 , and from $\sim 15 \%$ to $45 \%$ in model 2), but the corresponding mass fraction remains at $\sim 40 \%$. The volume filling factor of gas with temperatures $10^{4} \mathrm{~K}<T<10^{5} \mathrm{~K}$ decreases from $\sim 15 \%$ to $\sim 10 \%$ from $z=1$ to $z=0$ for both models, while the mass percentage drops from $\sim 25 \%$ to $\sim 10 \%$ for model 1 and from $\sim 20 \%$ to $\sim 10 \%$ for model 2 . For both models, the mass percentage in the temperatures $10^{5} \mathrm{~K}<T<5 \times 10^{5} \mathrm{~K}$ increases by $\sim 20 \%$ during the same period. These tendencies are anticipated on the basis of rather general considerations. Even though the amount of direct galaxy heating is negligible during the period

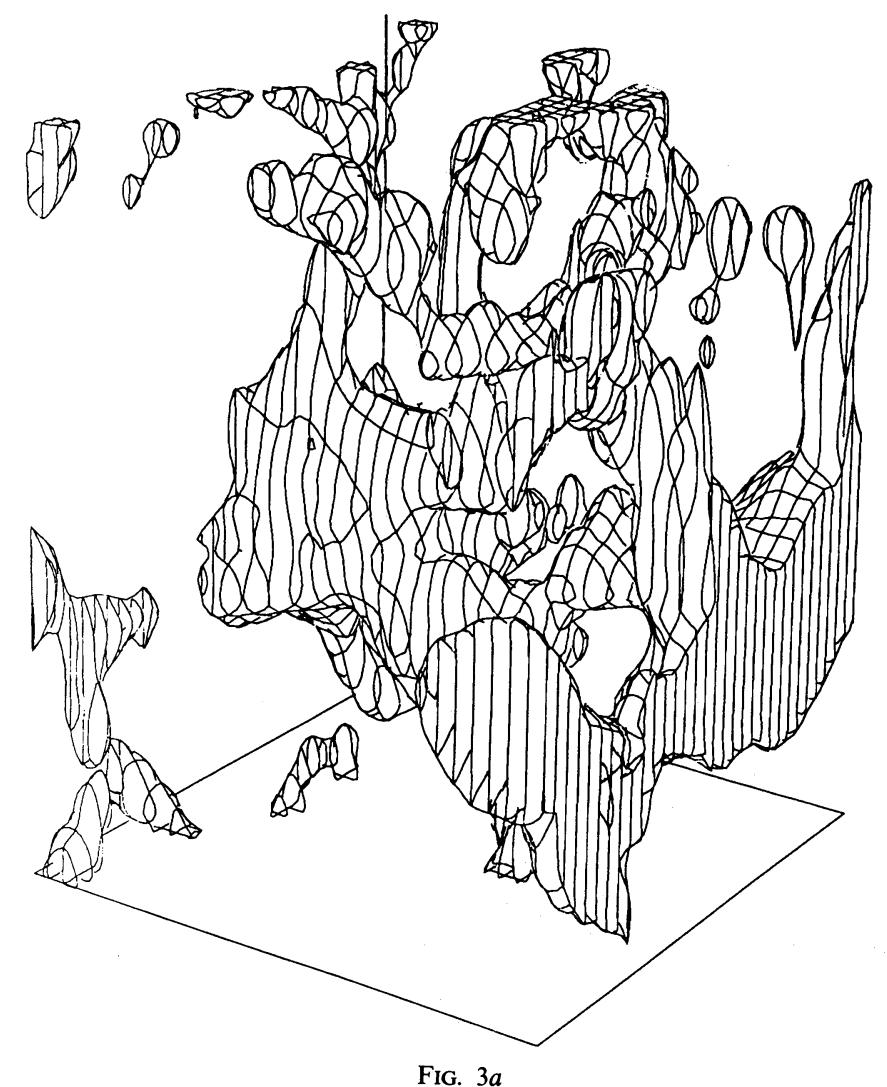

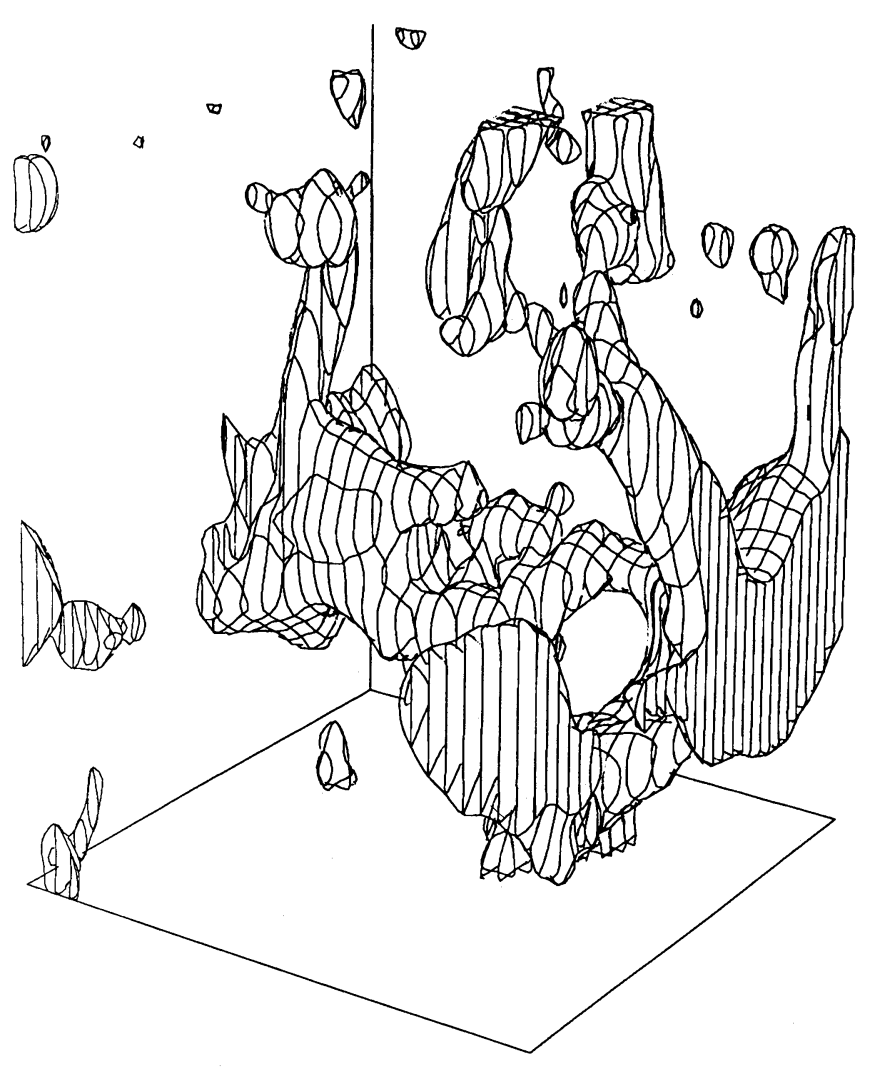

FIG. $3 b$

FIG. 3.- Equal density surfaces of the IGM at $(a) z=1$ and $(b) z=0$ chosen from the fourth run in model 1, the surfaces corresponding to a density twice that of the mean. No smoothing is employed. Note that the distribution is dominated by large connected structures. 


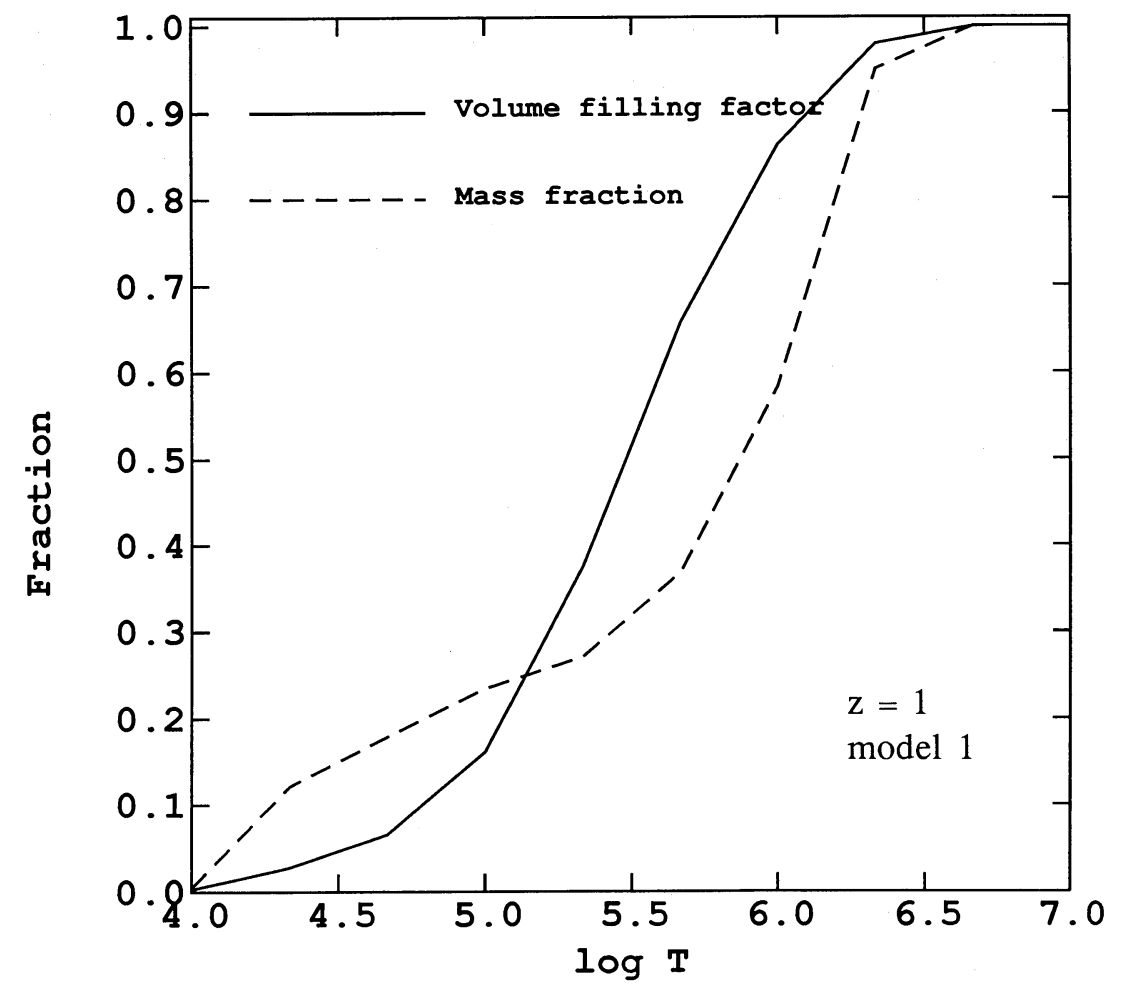

FIG. $4 a$

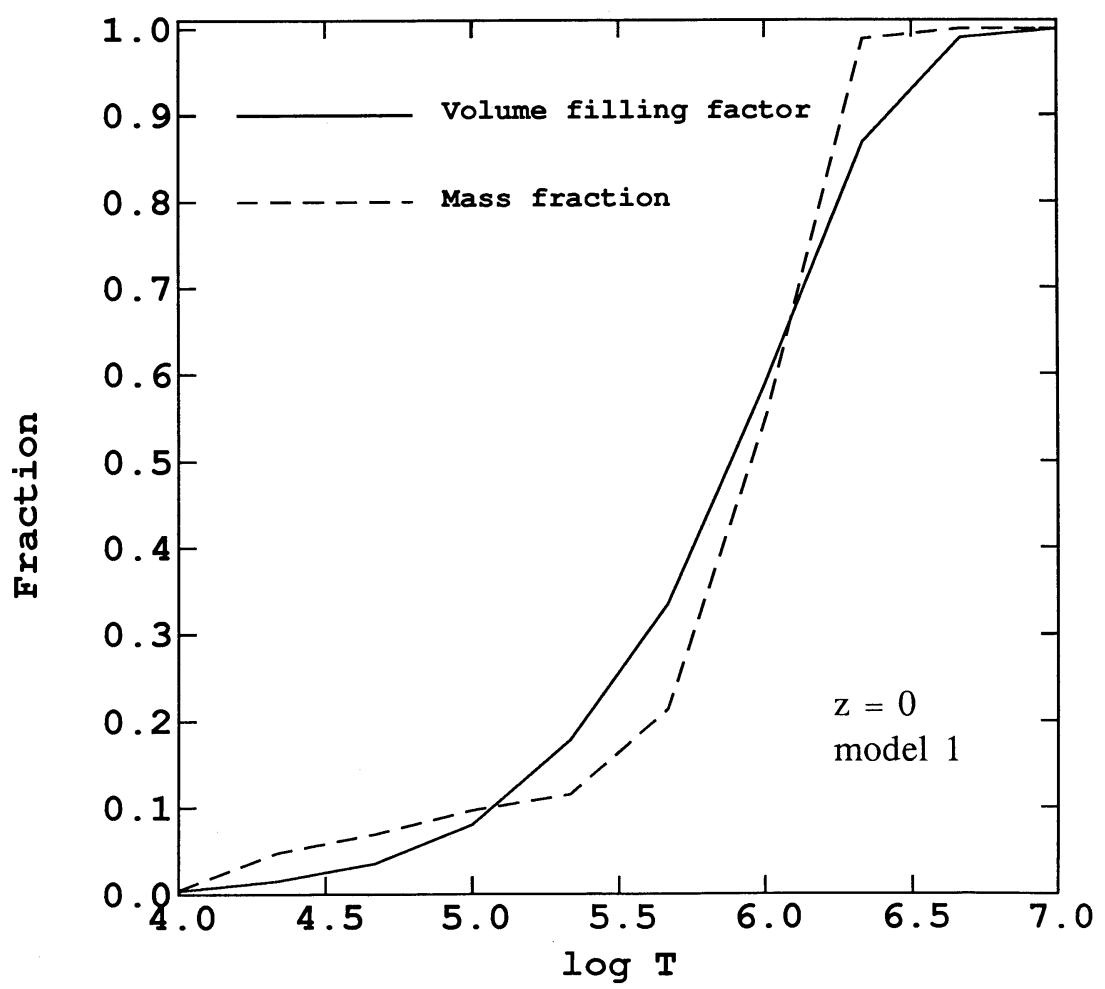

Fig. $4 b$

FIG. 4.-Cumulative volume filling factor (solid line) and mass fraction (dashed line) for the IGM are plotted at two epochs $(z=1$ and $z=0)$ for both models. The gas is predominantly ionized; there is a substantial amount of gas with temperatures above $10^{6} \mathrm{~K}$.

609 


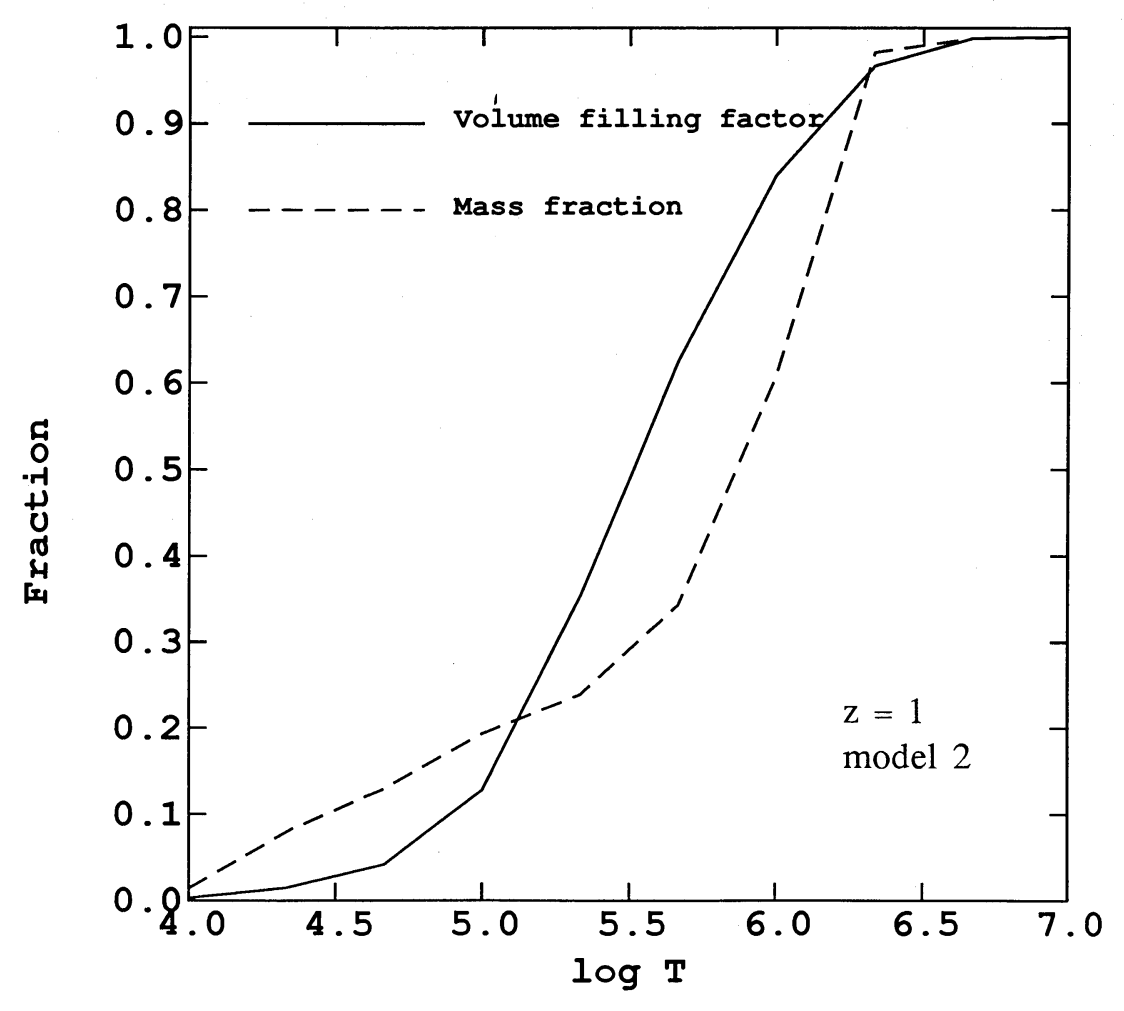

FIG. $4 c$

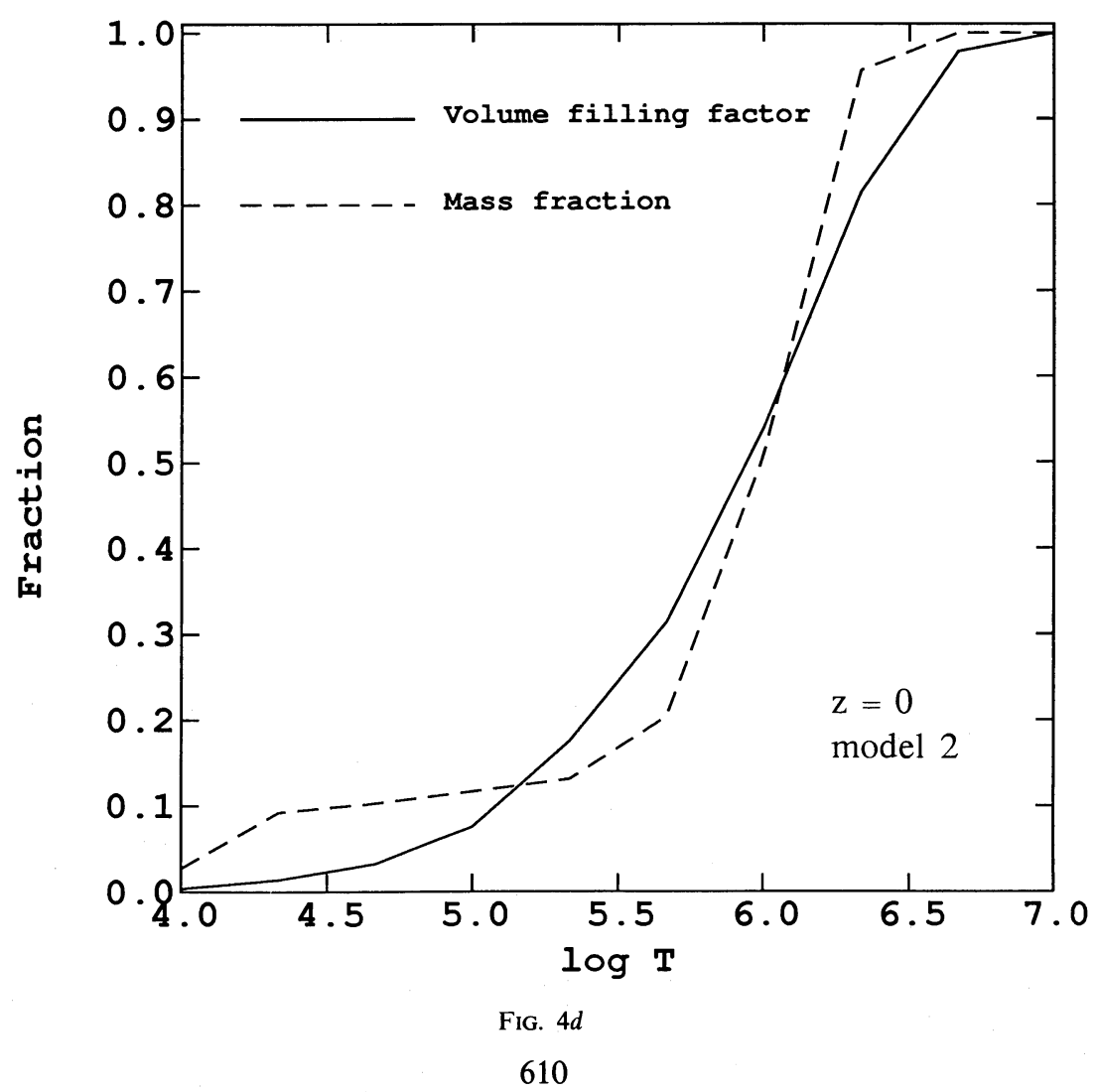


between $z=1$ and $z=0$, the gas continues to fall back into potential minimum regions from the more extended distribution created by galaxy heating during an earlier period. Thus part of the potential energy gained previously is converted into thermal energy as the kinetic energy of the gas is dissipated. For comparison we have also calculated a model for which the galaxy heating is absent. The result shows that $<10 \%$ of the gas has temperatures $T>10^{6} \mathrm{~K}, \sim 50 \%$ has temperatures $10^{4} \mathrm{~K}<T<3 \times 10^{4} \mathrm{~K}$, and $<10 \%$ has temperatures $T<10^{4} \mathrm{~K}$.

Additional information may be extracted by comparing the temperature and density distributions. As an example, Figure 5 shows two plots of equal-temperature surfaces, corresponding respectively to temperatures at $5 \times 10^{4}$ and at $3 \times 10^{6} \mathrm{~K}$ selected from run 4 of model 1 at the present epoch. The volume inside the surfaces contains gas with temperatures below $5 \times 10^{4} \mathrm{~K}$ in Figure $5 a$, and above $3 \times 10^{6} \mathrm{~K}$ in Figure $5 b$. Comparing Figure 5 with Figure 3, we note that cool gas is generally near large clumps of gas, whereas the hot gas is frequently located at the edges of the largest clumps.

\section{d) Hypothetical Line Profiles}

Since the observation of emission or absorption lines is the most promising way to probe the structure of the IGM, we computed a series of optically thin lines. For simplicity, we take lines of sight along one of the axes of the grid. For an optically thin line, its intensity is proportional to the amount of matter in the specified velocity interval. Gases of all temperatures are sampled. To improve the statistics, we sample
$4 \times 32$ cells along each line of sight. A resolution of $25 \mathrm{~km} \mathrm{~s}^{-1}$ is used; the results are not sensitive to the resolution.

Figure $6 a$ shows three examples of the computed line profiles chosen from different runs of model 1. Each line is calculated at $z=1$ and $z=0$. Figure $6 b$ shows the corresponding density, pressure, and temperature profiles (averaged over 4 cells). In computing these lines we have taken into account both the Hubble flow velocities and the peculiar velocities. From these few examples, it is apparent that a variety of profiles are possible, one two, or multiple components, for example, with a varying degree of velocity separations. Strong lines are frequently about 10 times as intense as weak lines, and they commonly coexist. In the case of profiles with multiple components there appears to be a tendency for them to cluster, but we do not have enough components to perform quantitative statistics.

In general, a given component in the profile comes from a distinctive region in the space unless there are other components nearby, because the Hubble velocity is generally larger than the peculiar velocity. For instance, at $z=0$ the Hubble velocity is $\sim 500 \mathrm{~km} \mathrm{~s}^{-1}$ across half the grid length, whereas the corresponding typical peculiar velocity of the model IGM is less than $200 \mathrm{~km} \mathrm{~s}^{-1}$. Thus the effect of velocity crowding is not important. A strong line simply means that the line of sight goes through a large condensation. We note that the profile along the same line of sight may change as the system evolves - the number of components may increase or decrease depending on whether the motion of the emitting gas enters or leaves the line of sight.

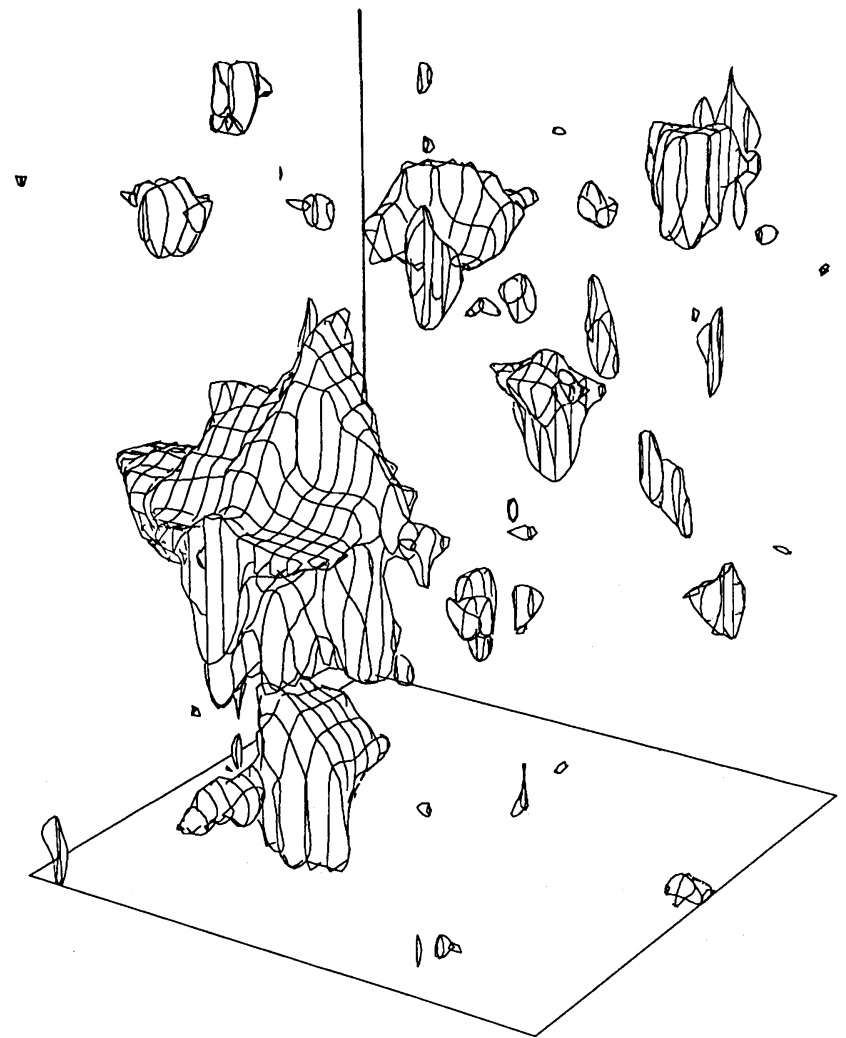

FIG. $5 a$

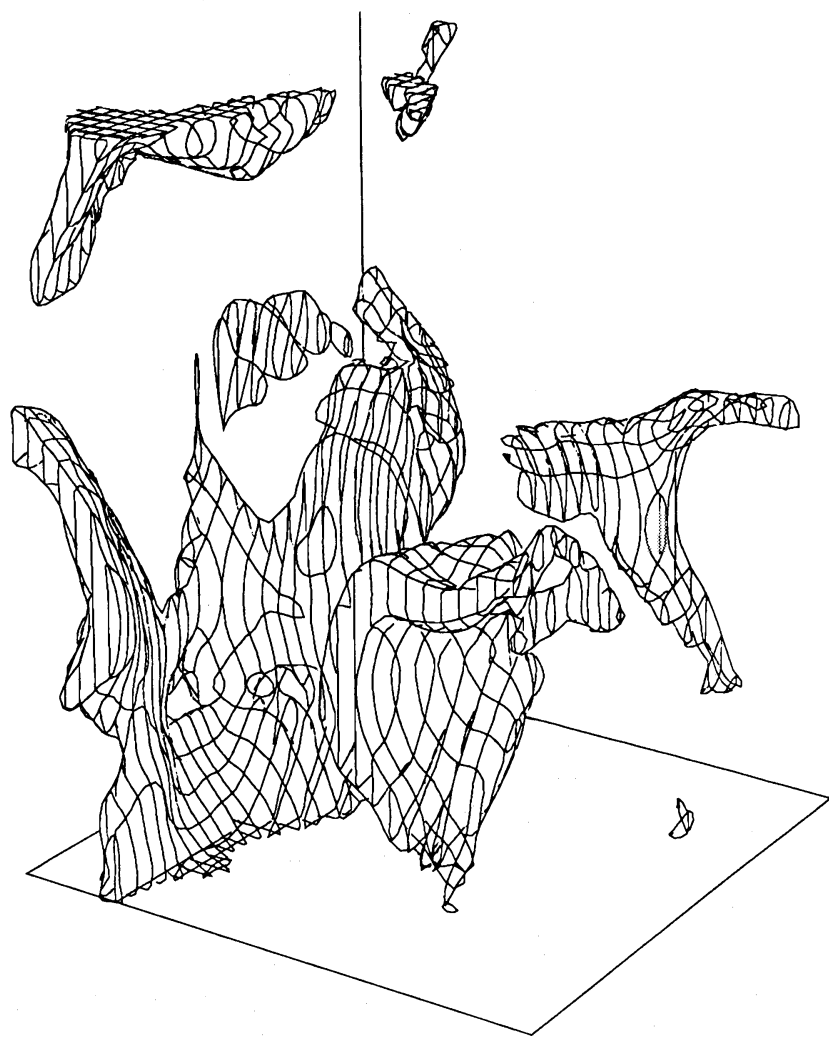

Fig. $5 b$

FIG. 5--Equal-temperature surfaces of the IGM at the present epoch, chosen from the fourth run in model 1 (same as those for Fig. 3). The volume inside the surface corresponds to gas with $(a) T<5 \times 10^{4} \mathrm{~K}$ and $(b) T>3 \times 10^{6} \mathrm{~K}$. The hot gas tends to be located at the peripheries of large condensations. 

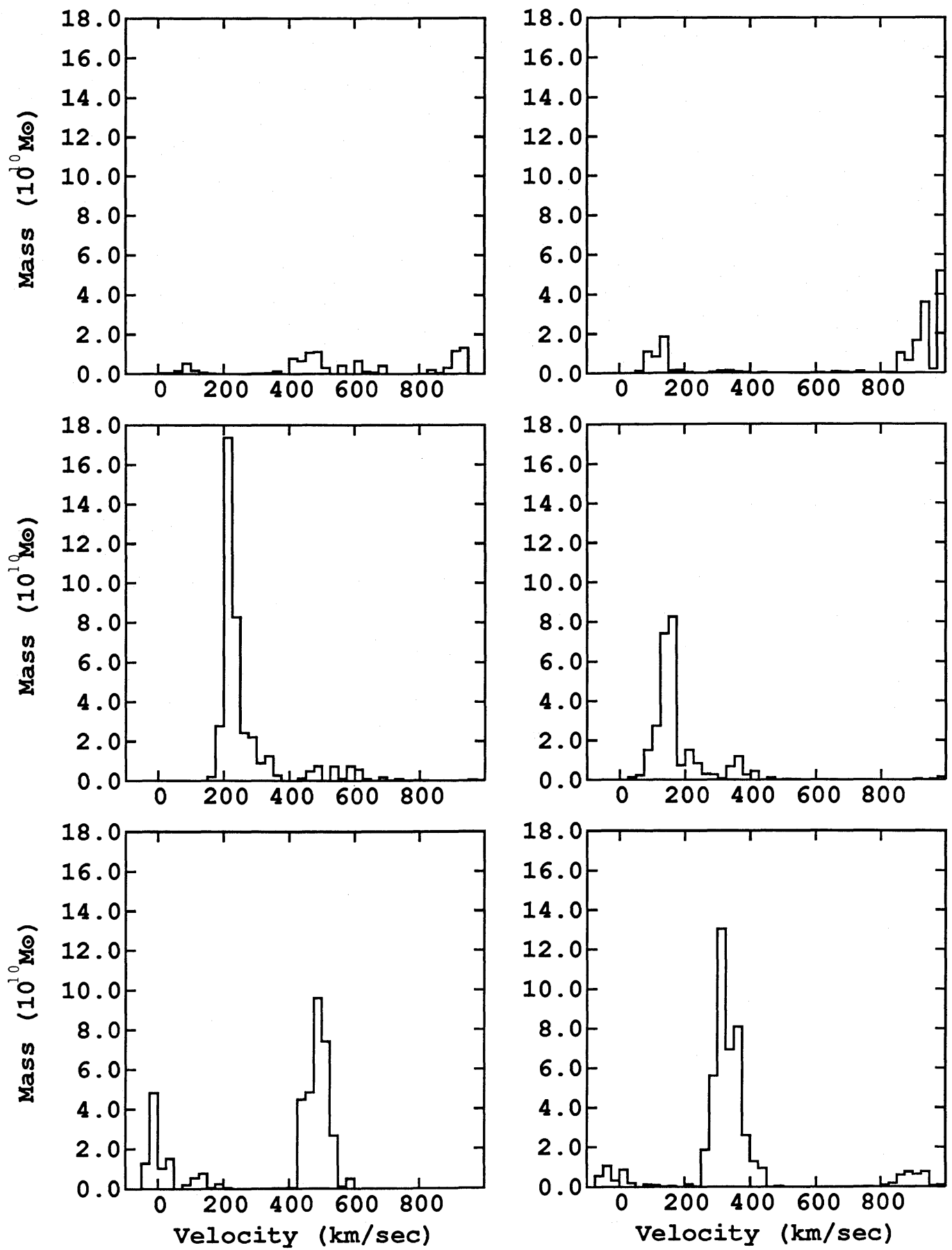

Fig. $6 a$

Fig. 6. - (a) Optically thin line profiles (sum over 4 cells) computed for model 1 at $z=1$ and $z=0$. Along a fixed line of sight, the profile changes because of the evolution in the large structure. (b) Corresponding density, pressure, and temperature profiles (averaged over 4 cells; in logarithmic scale). Density is in $10^{-30} \mathrm{~g} \mathrm{~cm}^{-3}$, pressure in $\mathrm{K} \mathrm{cm}^{-3}$, and temperature in $\mathrm{K}$.

The temperature profiles in Figure $6 b$ indicate that its variation is $\sim 1-2$ orders of magnitude, with most of the gas ionized. The typical temperature is slightly higher for the present epoch (see also the preceding section). Along the same lines of sight the pressure variation is frequently $2-3$ orders of magnitudes, pressures being usually higher in regions of higher densities. The IGM is not in a state of pressure equilibrium. We also note that the bottom pair of plots in Figure $6 b$ indicate that the clump located at $x \sim 12$ has approximately exponential density and pressure profiles. This gas appears to be in a state of hydrostatic equilibrium within the local potential of the dark matter.

\section{e) Power Spectrum and Two-Point Correlation Function}

The power spectrum is calculated by Fourier transforming the density fluctuations and binning to the nearest half-integral wavenumber. In the following discussion, the wavenumber $k$ 

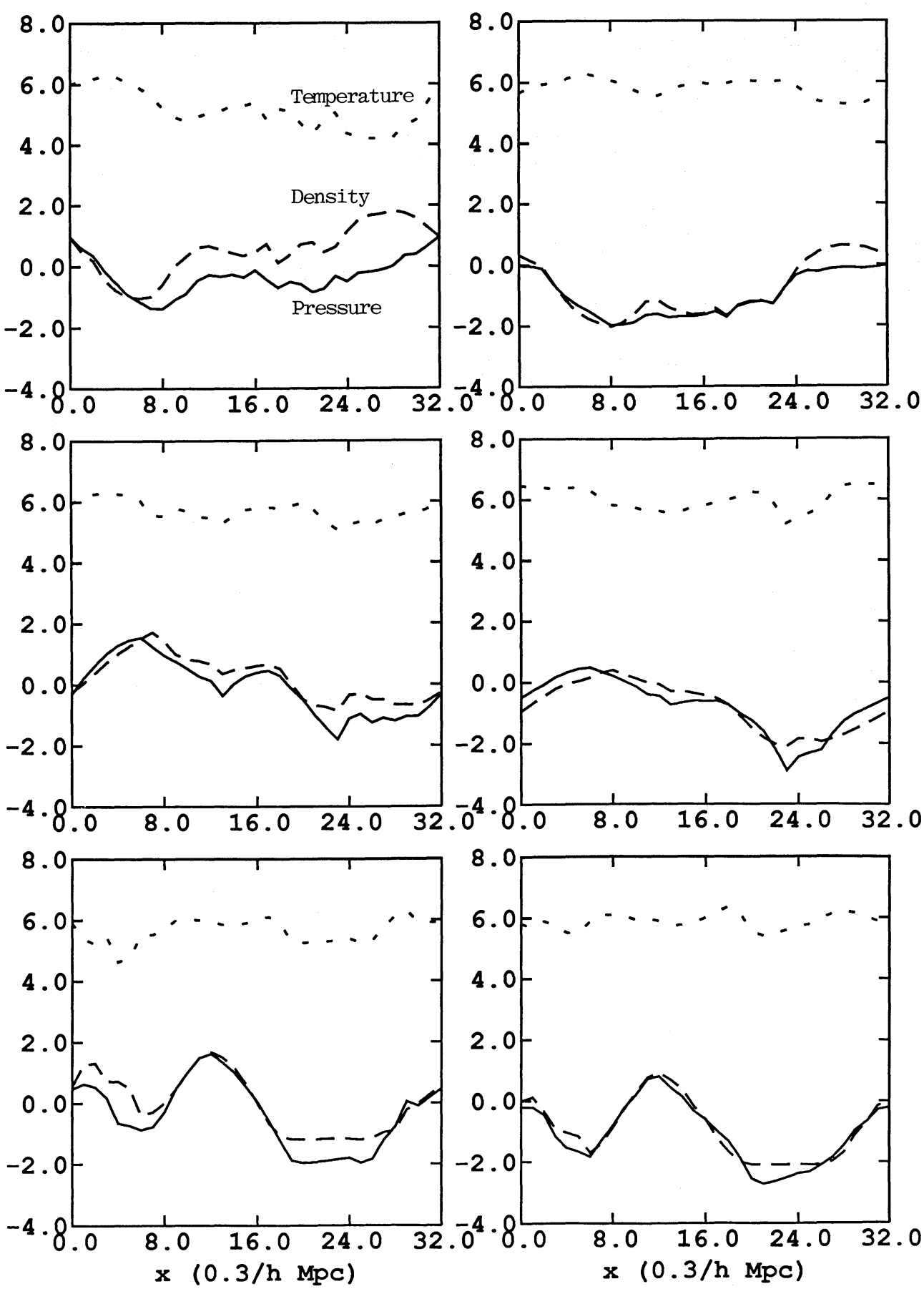

Fig. $6 b$

has units such that $k=1$ corresponds to a wavelength equal to the fundamental wavelength of the computational cube, i.e., $\lambda=32(\Delta x) \mathrm{Mpc}$, where $\Delta x$ is the cell size. The maximum frequency calculated is the Nyquist frequency, $k_{\mathrm{N}}=16$.

Figure 7 displays the evolution of the power spectrum of the IGM for both models. If the density fluctuation remains linear at the largest computational scales, the power spectrum for the dark matter at $k=1$ is expected to grow as $a^{2}$. Figure 7 shows that the growth of $\log P_{k=1}$ is $\sim 3.7$, while the linear theory predicts a growth of 4.6 for the dark matter. Thus the growth of the IGM is lower than that of the dark matter-perhaps not a surprise.

It is apparent from Figure 7 that in neither model does the power spectrum of the IGM evolve in a self-similar way. The error bars in model 2 are larger because of a greater dispersion in the number of galaxies formed in these four runs. On all scales and at all instants, the power in model 2 is consistently greater, especially pronounced on smaller scales. Such behavior is expected for three reasons. First, the effect of heating is smaller in model 2. Second, the pressure force is most effective 

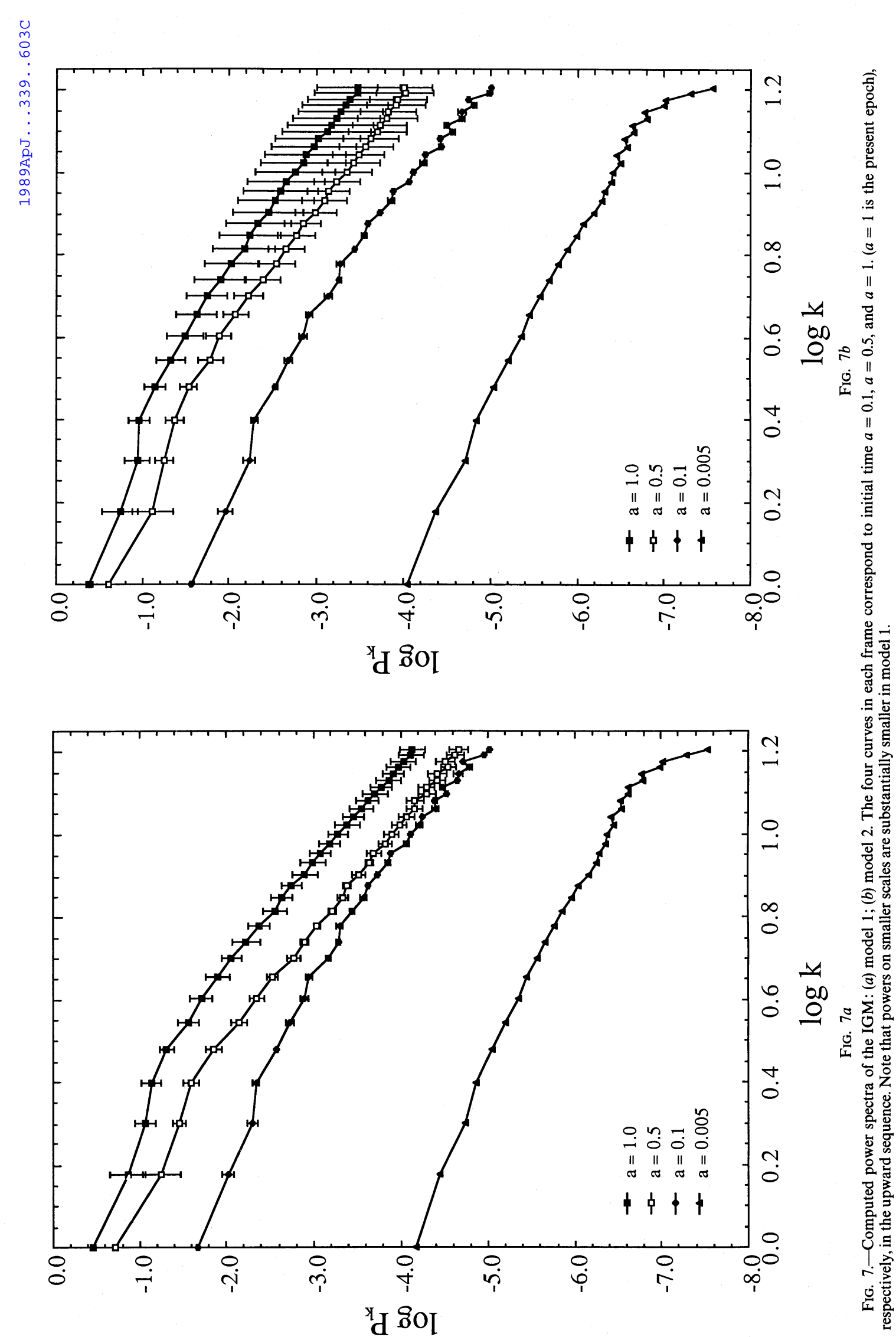

614 
in working against the gravity. Third, the growth period is longer in model 2. At the present epoch, the difference in the power spectra of the two models increases monotonically from a scale of $\sim 9.6 h^{-1} \mathrm{Mpc}$ down to a scale of $\sim 0.6 h^{-1} \mathrm{Mpc}$. At the short-wavelength end the power in model 2 is $\sim 4$ times that in model 1.

The corresponding two-point correlation functions are calculated by inverse Fourier transforming the power spectra and binning to the nearest half-integral normalized separation. Figure 8 shows the two-point correlation function for the IGM $\left(\xi_{\text {IGM }}\right)$. The difference between the two models is modest for separations $0.3 h^{-1} \mathrm{Mpc}<r<1.2 h^{-1} \mathrm{Mpc}$. The slopes of $\xi_{\text {IGM }}$ for both models approximately fit the observed galaxy-galaxy two-point correlation function $\left(\xi_{\mathrm{GG}}\right)$ for separations $0.75 h^{-1}$ Mpc $<r<2 h^{-1} \mathrm{Mpc}$. In general, $\xi_{\mathrm{IGM}}$ is expected to be different from $\xi_{\mathrm{GG}}$, since after formation galaxies are not subject to the pressure forces that affect the IGM. Still, it is possible that $\xi_{\text {IGM }}$ may give a fair estimate of $\xi_{\mathrm{GG}}$ for bright galaxies if the continued infall of gas leads to continued star formation.

\section{f) Peculiar Velocity Distribution}

The peculiar velocity distribution is computed using the densest 1000 cells. The result is not sensitive to the precise number of cells used. Three moments are computed: the first moment, $\left\langle v_{12 \|}\right\rangle$, is the mean relative peculiar velocity of pairs parallel to the direction connecting the pair, and the two second moments, $\left\langle v_{12 \|}^{2}\right\rangle^{1 / 2}$ and $\left\langle v_{12 \perp}^{2}\right\rangle^{1 / 2}$, are the rms relative peculiar velocities parallel and perpendicular, respectively, to the direction connecting the chosen pair of cells. Our results are shown in Figure 9.

The moment $\left\langle v_{12 \|}\right\rangle$ of the IGM is qualitatively similar for the two models at the epochs $z=1$ and $z=0$. It increases gradually for separations $0.3 h^{-1} \mathrm{Mpc}<r<2.4 h^{-1} \mathrm{Mpc}$ and then drops to zero at separations $\sim 5 h^{-1} \mathrm{Mpc}$. The peak value is $\sim 110 \mathrm{~km} \mathrm{~s}^{-1}$ at $z=1$ and drops slightly to $\sim 90-100 \mathrm{~km}$ $\mathrm{s}^{-1}$ by $z=0$. Also, the first moment always falls below the Hubble line. At $z=1,\left\langle v_{12 \|}^{2}\right\rangle^{1 / 2}$ is larger than $\left\langle v_{121}^{2}\right\rangle^{1 / 2}$ for all separations for both models, but at $z=0\left\langle v_{12 \|}^{2}\right\rangle^{1 / 2}$ becomes smaller than $\left\langle v_{12 \perp}^{2}\right\rangle^{1 / 2}$ for separations larger than $\sim 2.4 h^{-1} \mathrm{Mpc}$. The predominance of a larger $\left\langle v_{12 \|}^{2}\right\rangle^{1 / 2}$ is consistent with the fact that the gas, after a phase of expansion, collapses back to regions of gravitational potential minimum. As the process subsides, the difference between the two second moments narrows.

\section{SUMMARY AND DISCUSSION}

The model results may be summarized as follows: (1) The growth of density fluctuations on small scales in the gas component is substantially reduced during the period of galaxy heating. (2) Large connected structures dominate the distribution of the model IGM. (3) The gas is mostly ionized. Less than $1 \%$ of the gas has temperatures below $10^{4} \mathrm{~K}$, whereas $\sim 40 \%$ has temperatures above $10^{6} \mathrm{~K}$. The amount of moderately hot gas $\left(10^{5} \mathrm{~K}<T<5 \times 10^{5} \mathrm{~K}\right)$ may increase as a result of gas flows converging back to local gravitational potential minima. (4) Pressure equilibrium is absent in the model IGM. The variation in the pressure is typically 10 times that in the temperature; pressures are usually higher in high-density regions. Condensed gas is confined by the gravity of the dark matter, except during the very short period of galaxy heating. (5) Optically thin lines show a variety of profiles. (6) At late times, the power spectrum of the model IGM is substantially depressed at short wavelengths. The two-point correlation function of the model IGM fits the observed galaxy-galaxy two-point correlation function for separations $0.3 h^{-1} \mathrm{Mpc}<r<2.4 h^{-1} \mathrm{Mpc}$. (7) The first moment of the peculiar velocity distribution increases gradually from a small separation to a separation of $2.4 h^{-1} \mathrm{Mpc}$, and then drops fairly rapidly to zero at a separation of $\sim 5 h^{-1} \mathrm{Mpc}$. The parallel component of the second moment is generally larger than that of the perpendicular component. (8) These results seem insensitive to cosmological parameters. They apply to both models studied.

Because of the uncertainties inherent in the use of a pressureless fluid approximation for the CDM when the evolution enters the highly nonlinear regime, the model results for the IGM should be considered more reliable. Nevertheless, we will summarize the main qualitative features concerning the results of the CDM component. These qualitative features are probably correct. (1) The distribution of the CDM is also dominated by large connected structures, but the distribution is clumpier than that of the IGM. Large structures are reasonably well correlated between the two components, but small clumps of dark matter frequently are not associated with any IGM. (2) The growth of a density fluctuation is more rapid in the dark matter. At small wavelength the density fluctuations in the CDM are much more pronounced than those in the IGM. (3) The fit of the two-point correlation function of the dark matter to the observed galaxy-galaxy two-point correlation function is less satisfactory than that of the IGM. The qualitative behavior of the velocity distributions of the dark matter is similar to that of the IGM, but the numerical values are larger.

A notable feature in the model IGM is the absence of a pressure equilibrium. The fact that at late times the bulk of the gas is converging toward regions of abundant dark matter indicates that the IGM is confined by gravity of the CDM, on the scale of a few megaparsecs. Confinement by self-gravity cannot be ruled out by our models, since gravity due to gas is not computed explicitly, but it appears unlikely. The sizes of individual gas condensations are typically far larger than that of the Ly $\alpha$ clouds inferred from observations, and therefore the former cannot be compared directly with the latter. Indeed, the model IGM distribution is dominated by large connected structures. It is, however, tempting to think that Ly $\alpha$ lines might originate from smaller condensations within these large structures. In this view, one might expect clustering of Ly $\alpha$ lines on scales up to a few $100 \mathrm{~km} \mathrm{~s}^{-1}$, depending on the lines of sight, if we assume that Ly $\alpha$ clouds are distributed approximately uniformly within these larger structures. Observationally there is some indication of weak clustering for the Ly $\alpha$ clouds on a scale of $\sim 100-200 \mathrm{~km} \mathrm{~s}^{-1}$ (Crotts 1988; Webb 1987). Along the same line of reasoning, we might also anticipate the existence of large velocity intervals (several hundred $\mathrm{km} \mathrm{s} \mathrm{s}^{-1}$; see Fig. 6) without $\mathrm{Ly} \alpha$ absorption lines, again depending on the line of sight. However, a different statistical interpretation of the distribution of the Ly $\alpha$ clouds is proposed by Ostriker, Bajtlik, and Duncan (1988). Last, we should keep in mind that the distribution of Ly $\alpha$ clouds, as deduced from observations of a relatively small number of quasars $(\sim 10)$, may not represent the true distribution of the IGM.

An important constraint of the IGM is the Gunn-Peterson test. Our current result suggests that the fraction of the neutral hydrogen is very small. Since the present model does not compute the ionization structure in the IGM, it would be premature to make any quantitative comparison. A better method of computing the dynamics of the dark matter will 

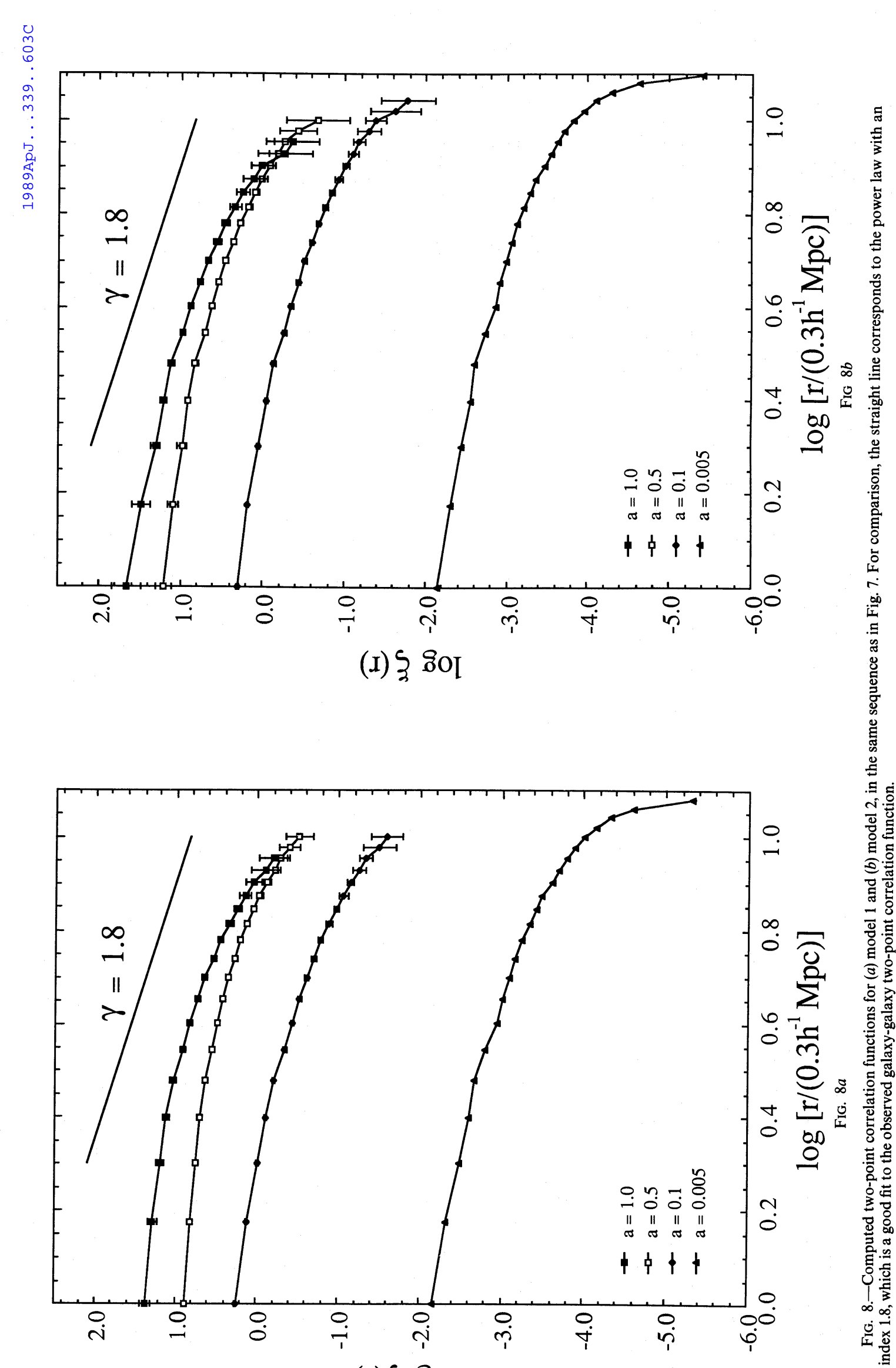

(I) รุ 80 


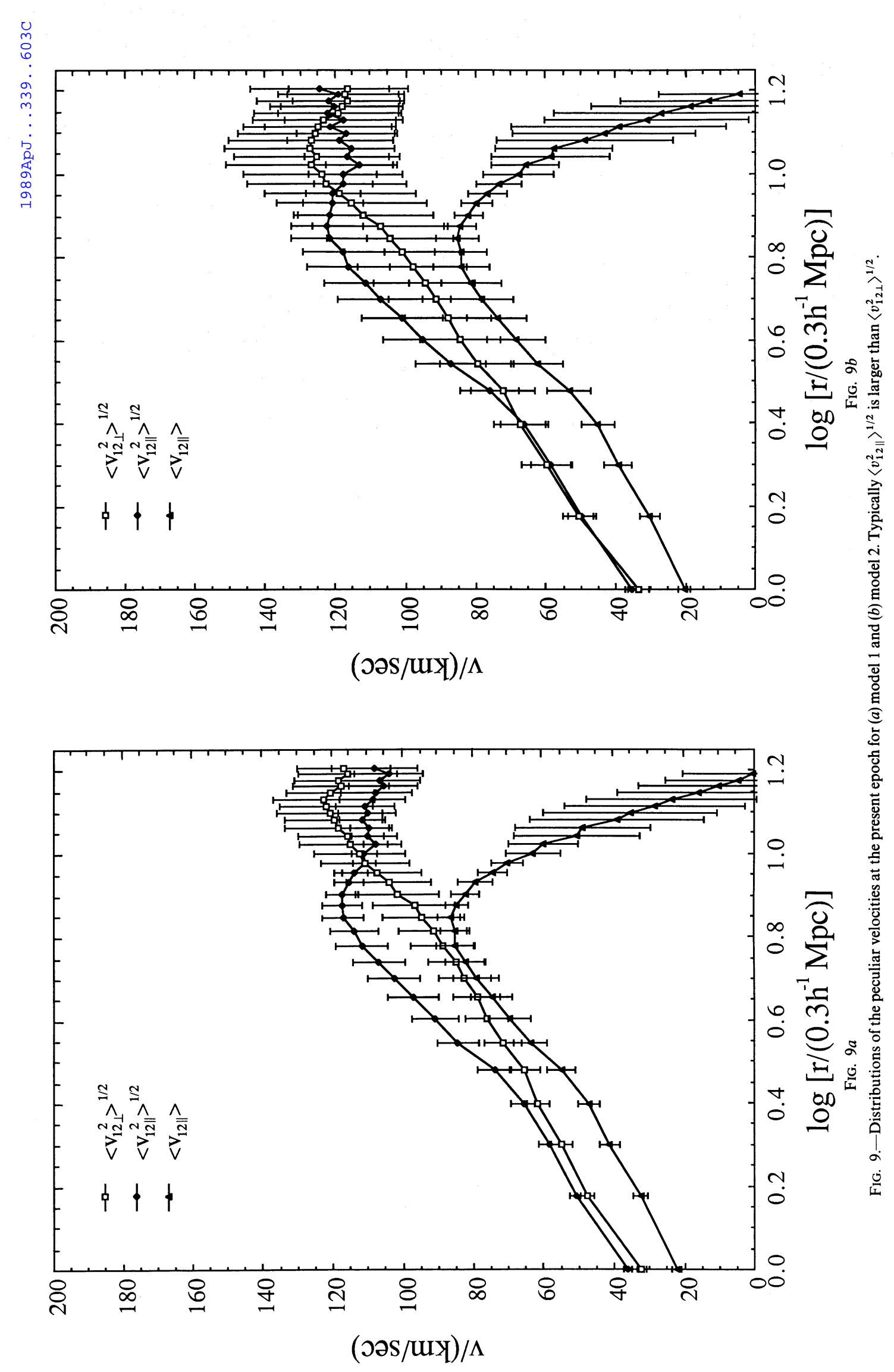

617 
improve the present model significantly. However, it appears that even with all its simplifications, our model is still useful for an exploratory study.
We would like to thank A. Crotts for discussions on the Ly $\alpha$ absorption lines. This work is supported in part by National Science Foundation grant AST8451736.

\section{REFERENCES}

Black, J. H. 1981, M.N.R.A.S., 197, 553.

Peebles, P. J. E. 1987, Ap. J. 317, 576

Rees, M. J. 1986, M.N.R.A.S., 218, 25P.

Ryu, D., and Vishniac, E. T. 1988, preprint.

Salmon, J., and Hogan, C. 1986, M.N.R.A.S., 221, 93.

Sanders, R. H., and Prendergast, K. H. 1974, Ap. J., 188, 489.

Sargent, W. L., Young, P. J., Boksenberg, A., and Tytler, D. 1980, Ap. J. Suppl., $42,41$.

Vishniac, E. T. 1983, Ap. J., 274, 152.

Vishniac, E. T., and Bust, G. S. 1987, Ap. J., 319, 14.

Vishniac, E. T., Ostriker, J. P., and Bertschinger, E. 1985, Ap. J., 291, 399.

Webb, J. K. 1987, in IAU Symposium 124, Observational Cosmology, ed. A. Hewitt, G. Burbidge, and L. Z. Fang (Dordrecht: Reidel), p. 803

Weedman, D. W. 1986, Quasar Astronomy (Cambridge: Cambridge University Press).

White, S. D. M. 1986, in Nearly Normal Galaxies, ed. S. M. Faber (New York: Springer-Verlag)

White, S. D. M., Frenk, C., Davis, M., and Efstathiou, G. 1987, Ap. J., 313, 505.

Melott, A. L., Einasto, J., Saar, E., Suisalu, I., Klypin, A. A., and Shandarin, S. F. 1983, Phys. Rev. Letters, 51, 935.

Ostriker, J. P., Bajtlik, S., and Duncan, R. C. 1988, Ap. J. (Letters), 327, L35.

Ostriker, J. P., and Ikeuchi, S. 1983, Ap. J. (Letters), 268, L63.

Peebles, P. J. E. 1982, Ap. J. (Letters), 263, L1.

Wei-Hwan Chiang, Dongsu Ryu, and Ethan T. VishniaC: Astronomy Department, University of Texas, Austin, TX 78712-1083 